\title{
Misidentification of Bordetella bronchiseptica as Bordetella pertussis using a newly described real- time PCR targeting the pertactin gene
}

\author{
Correspondence \\ Karen B. Register \\ kregiste@nadc.ars.usda.gov
}

Received 13 July 2007

Accepted 18 August 2007

\author{
Karen B. Register and Tracy L. Nicholson
}

\author{
Respiratory Diseases of Livestock Research Unit, USDA/Agricultural Research Service/National \\ Animal Disease Center, Ames, IA 50010, USA
}

\begin{abstract}
Recently, a real-time PCR (RT-PCR) assay based on sequence from the gene for pertactin was proposed for identification of Bordetella pertussis. Here, it is reported that the $B$. pertussis pertactin gene sequence for the region that encompasses the RT-PCR probe and primers is nearly identical to that of many Bordetella bronchiseptica strains of human and avian origin. Additionally, it is demonstrated that such strains are erroneously identified as $B$. pertussis using the RT-PCR assay. These data suggest that the use of the assay without confirmatory testing may result in erroneous identification of a significant proportion of human isolates of $B$. bronchiseptica as $B$. pertussis.
\end{abstract}

\section{INTRODUCTION}

Bordetella pertussis is the aetiologic agent of whooping cough, an acute respiratory disease that occurs exclusively in humans. A rapid, sensitive and specific method for the identification of $B$. pertussis is crucial for the effective management of outbreaks and to monitor prevalence. The repetitive element IS481 is frequently targeted for rapid detection of $B$. pertussis by PCR (Riffelmann et al., 2005). While assays based on this approach are highly sensitive, the presence of IS481 in other species of Bordetella compromises their specificity (Diavatopoulos et al., 2005; Register \& Sanden, 2006; Riffelmann et al., 2005; van der Zee et al., 1997). Recently, a novel real-time PCR (RTPCR) assay that targets the gene for pertactin, a Bordetella adhesin and virulence factor, was proposed as an alternative (Vincart et al., 2007). Although the authors concluded that the assay alleviates problems related to cross-reactivity with Bordetella species other than pertussis, only single isolates of other species appear to have been evaluated. The goals of the present study were to determine the sequence of the relevant region of the pertactin gene from a large number of Bordetella bronchiseptica strains, including several of human origin, for comparison with the sequence of $B$. pertussis, and to evaluate the potential for false-positive results using the pertactin RT-PCR.

Abbreviation: RT-PCR, real-time PCR.

The GenBank/EMBL/DDBJ accession numbers for the Bordetella sequences determined in this paper are EU031919-EU032001.

A table showing $B$. bronchiseptica isolates used for sequence comparisons is available as supplementary data with the online version of this paper.

\section{METHODS}

Bacterial isolates. This study includes data derived from 88 isolates of B. bronchiseptica, obtained from at least 11 different host species. Initial identification of isolates was based on colony morphology and standard biochemical testing, and was confirmed by subsequent ribotype analysis (Register et al., 1997). Information related to individual isolates is available in Supplementary Table S1. B. pertussis Tohama I was used as a positive control in the pertactin RT-PCR. All isolates were grown at $37{ }^{\circ} \mathrm{C}$ for $36 \mathrm{~h}$ (B. bronchiseptica) or $72 \mathrm{~h}(B$. pertussis) on Bordet-Gengou agar supplemented with $10 \%$ sterile, defibrinated sheep's blood.

PCR and sequence analysis. Chromosomal DNA was purified with a commercially available kit (Gentra Systems) and quantified with PicoGreen (Molecular Probes). The Primer Design module of Vector NTI Advance (Invitrogen) was used to select PCR primers for amplification of the pertactin gene region of interest (forward, $5^{\prime}$ GATGATGCGTCGCTGTAACA-3'; reverse, 5' -AGGGTGCCGATATGCAAGCC-3'). PCR was carried out using $100 \mathrm{ng}$ purified DNA and the reaction components and cycling conditions described previously (Register, 2004). Five microlitres of each reaction was analysed by agarose gel electrophoresis in 3:1 NuSieve (Cambrex BioScience) containing $0.5 \mu \mathrm{g}$ ethidium bromide $\mathrm{ml}^{-1}$. Amplicons were purified using spin columns (Qiagen), and were sequenced directly by fluorescence-based cycle sequencing with AmpliTaq and BigDye Terminators on an ABI 377 sequencer, at the National Animal Disease Center Genomics Unit. Sequences were analysed using Vector NTI Suite software.

RT-PCR. RT-PCR was carried out using purified DNA from the strains indicated according to the method described elsewhere (Vincart et al., 2007). The detection rate of this assay has been determined to be 90,100 and $100 \%$ at $10 \log _{10}\left(\right.$ copies $\mathrm{ml}^{-1}$ ) for $B$. pertussis strains ATCC 9797, ATCC 9340 and Bord 201, respectively. Therefore, $10 \log _{10}\left(\right.$ copies $\mathrm{ml}^{-1}$ ) was chosen as the detection limit for all strains evaluated in this report. 


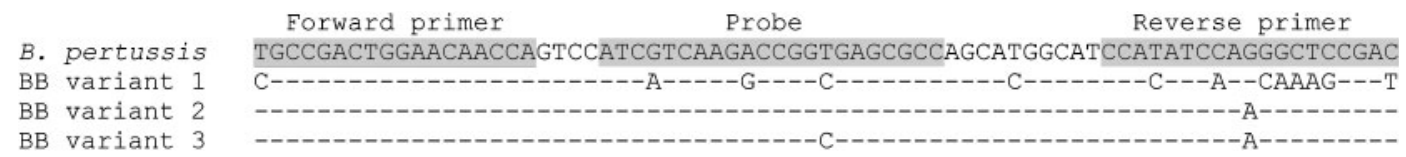

Fig. 1. Alignment of the $B$. pertussis gene comprising covering the pertactin RT-PCR primers and probe with those of the three $B$. bronchiseptica (BB) sequence variants. Dashes represent conserved bases; substitutions are indicated by the appropriate letter. Although not depicted here, the corresponding sequences of the four currently available strains of $B$. parapertussis are identical to that of $B$. bronchiseptica variant 1 .

\section{RESULTS AND DISCUSSION}

The earlier study that evaluated the performance characteristics of the pertactin RT-PCR included a single, unnamed isolate each of B. bronchiseptica and Bordetella parapertussis (Vincart et al., 2007). A search of the GenBank database identified five B. bronchiseptica isolates and four $B$. parapertussis isolates for which the pertactin gene sequence corresponding to the RT-PCR probe and primers is available. We additionally determined the corresponding sequence from 83 strains of B. bronchiseptica selected from the National Animal Disease Center collection, representing isolates from humans and a variety of other host species. Alignment of the DNA sequences from the $88 \mathrm{~B}$. bronchiseptica isolates revealed three unique variants, none of which is identical to the sequence reported for $B$. pertussis (Fig. 1). Variant 1 was found with the highest frequency in the $B$. bronchiseptica strains examined $(64.8 \%)$ and in all but one host species of origin (Table 1), as well as in the four $B$. parapertussis strains evaluated (GenBank accession nos BD409974, AX418063, X54547 and BX640426). One or more base mismatches are evident with respect to both the forward and reverse RT-PCR primers as well as the probe sequence proposed for identification of $B$. pertussis. Variants 2 and 3 were found in 22.7 and $12.5 \%$ of B. bronchiseptica isolates, respectively, with the majority detected in strains of human or avian origin. Variants 2 and 3 contain only one or two base mismatches compared to the corresponding $B$. pertussis sequence.

These data suggest that some strains of B. bronchiseptica (specifically, those with sequence variants 2 or 3 ) may be misidentified as $B$. pertussis using the proposed pertactin RT-PCR (Vincart et al., 2007). To address this possibility, the assay was carried out using purified DNA from $B$. pertussis and from two representatives each of $B$. bronchiseptica sequence variants 1,2 and 3 . As shown in Table 2, B. bronchiseptica strains St Louis (variant 2), MBORD839 (variant 2), MO211 (variant 3) and F4563 (variant 3) were falsely identified as B. pertussis by the proposed pertactin RT-PCR assay. As expected, $B$. bronchiseptica strains RB50 and KM22 (both variant 1) were not identified as $B$. pertussis.

It has been suggested that the pertactin RT-PCR alleviates the problem of cross-reactivity with Bordetella species other than pertussis and may not require a confirmatory test (Vincart et al., 2007). The specificity of the assay is purported to be superior to that of PCRs that target the repetitive insertion sequence IS481. However, these conclusions appear to have been based on the analysis of only a single strain from each of the other Bordetella species tested. Our results indicate that the pertactin RT-PCR is likely to incorrectly identify a large proportion of human $B$. bronchiseptica isolates as B. pertussis, since $43.5 \%$ of the human isolates examined here possess sequence demonstrated to return a false-positive result. In fact, IS481-based assays may provide a specificity superior to that of the pertactin RT-PCR, since the insertion element has so far not been detected in any human $B$. bronchiseptica isolate (Diavatopoulos et al., 2005; Register \& Sanden, 2006; van der Zee et al., 1997).

Other investigators have recently analysed the region of the pertactin gene that encodes the extracellular domain from $50 \mathrm{~B}$. bronchiseptica isolates different from those in the present study (Diavatopoulos et al., 2006). Those sequences overlap the region evaluated here, but do not include the $4 \mathrm{bp}$ at the extreme $5^{\prime}$ end. All but a single sequence, obtained from a seal isolate, match those reported here for variant 1,2 or 3 . The unique sequence in the seal isolate is identical to that of variant 1 , except for an additional base

Table 1. Distribution of $B$. bronchiseptica pertactin RT-PCR sequence variants among host species of origin

\begin{tabular}{|lccccccccccccc}
\hline $\begin{array}{l}\text { Sequence } \\
\text { variant }\end{array}$ & \multicolumn{10}{c|}{ Host species } \\
\cline { 2 - 12 } & Dog & Human & Turkey/avian & Pig & Horse & Guinea pig & Rabbit & Seal & Sea otter & Koala & Leopard Unknown \\
\hline 1 & 25 & 13 & 0 & 8 & 1 & 2 & 1 & 1 & 1 & 1 & 1 & 3 \\
2 & 1 & 4 & 15 & 0 & 0 & 0 & 0 & 0 & 0 & 0 & 0 & 0 \\
3 & 0 & 6 & 3 & 1 & 1 & 0 & 0 & 0 & 0 & 0 & 0 \\
\end{tabular}


Table 2. Pertactin RT-PCR results using representative $B$. bronchiseptica isolates

\begin{tabular}{|lc|}
\hline Strain* & Ct value (cycle) \\
\hline B. pertussis Tohama I & 22.73 \\
B. bronchiseptica RB50 (variant 1) & Undetermined \\
B. bronchiseptica KM22 (variant 1) & Undetermined \\
B. bronchiseptica St Louis (variant 2) & 19.90 \\
B. bronchiseptica MBORD839 (variant 2) & 19.21 \\
B. bronchiseptica MO211 (variant 3) & 26.38 \\
B. bronchiseptica F4563 (variant 3) & 22.59 \\
\hline
\end{tabular}

${ }^{\star}$ All strains were evaluated using $10 \log _{10}\left(\right.$ copies $\left.\mathrm{ml}^{-1}\right)$.

mismatch relative to $B$. pertussis in the region comprising the RT-PCR probe. Based on our RT-PCR results with variant 1 B. bronchiseptica strains, isolates with the novel sequence are not likely to be mistakenly identified as $B$. pertussis, although we have not directly tested this assumption. Due to the absence of sequence data for the bases corresponding to the extreme $5^{\prime}$ end of the forward pertactin RT-PCR primer, it is not possible to conclude with certainty whether additional variants might exist among the remaining $49 \mathrm{~B}$. bronchiseptica strains examined elsewhere (Diavatopoulos et al., 2006).

Given the lack of specificity demonstrated by the results reported here, the proposed pertactin RT-PCR cannot be recommended for identification of $B$. pertussis in the absence of additional confirmatory testing. Further efforts are required to identify highly sensitive and specific targets suitable for definitive identification of B. pertussis by PCR.

\section{ACKNOWLEDGEMENTS}

The authors thank Michael Mullins and Gwen Nordholm for expert technical assistance.

\section{REFERENCES}

Diavatopoulos, D. A., Cummings, C. A., Schouls, L. M., Brinig, M. M., Relman, D. A. \& Mooi, F. R. (2005). Bordetella pertussis, the causative agent of whooping cough, evolved from a distinct, human-associated lineage of B. bronchiseptica. PLoS Pathog 1, e45.

Diavatopoulos, D. A., Hijnen, M. \& Mooi, F. R. (2006). Adaptive evolution of the Bordetella autotransporter pertactin. J Evol Biol 19, 1931-1938.

Register, K. B. (2004). Comparative sequence analysis of Bordetella bronchiseptica pertactin gene (prn) repeat region variants in swine vaccines and field isolates. Vaccine 23, 48-57.

Register, K. B. \& Sanden, G. N. (2006). Prevalence and sequence variants of IS481 in Bordetella bronchiseptica: implications for IS481-based detection of Bordetella pertussis. J Clin Microbiol 44, 4577-4583.

Register, K. B., Boisvert, A. \& Ackermann, M. R. (1997). Use of ribotyping to distinguish Bordetella bronchiseptica isolates. Int J Syst Bacteriol 47, 678-683.

Riffelmann, M., Wirsing von Konig, C. H., Caro, V. \& Guiso, N. (2005). Nucleic acid amplification tests for diagnosis of Bordetella infections. J Clin Microbiol 43, 4925-4929.

van der Zee, A., Mooi, F., Van Embden, J. \& Musser, J. (1997). Molecular evolution and host adaptation of Bordetella spp.: phylogenetic analysis using multilocus enzyme electrophoresis and typing with three insertion sequences. J Bacteriol 179, 6609-6617.

Vincart, B., De Mendonca, R., Rottiers, S., Vermeulen, F., Struelens, M. J. \& Denis, O. (2007). A specific real-time PCR assay for the detection of Bordetella pertussis. J Med Microbiol 56, 918-920. 